Check for updates

Cite this: Chem. Commun., 2020, 56,1231

Received 4th October 2019,

Accepted 18th December 2019

DOI: $10.1039 / c 9 c c 07805 c$

rsc.li/chemcomm

\section{A facile analytical method for reliable selectivity examination in cofactor NADH regeneration $\dagger$}

\author{
Tony Saba, Joseph W. H. Burnett, Jianwei Li, Panagiotis N. Kechagiopoulos (D) and \\ Xiaodong Wang ${ }^{*} *$
}

This study demonstrates a novel method to quantify selective (1,4-NADH) and unselective products (1,2- and 1,6-NADH) in NADH regeneration using combined UV-Vis spectroscopy and biological assays. The validity of the proposed method was tested in the $\mathrm{Pt} / \mathrm{C}$ promoted $\mathrm{NAD}^{+}$hydrogenation using hydrogen as reducing agent.

1,4-NADH is a valuable cofactor that is used in tandem with enzymes (i.e. oxidoreductases) in the chemical and pharmaceutical industries for the manufacture of intermediates and drugs. Notable examples of these products include the Lipitor (atorvastatin), the antilipemic agent ZETIA (ezetimibe), the HIV protease inhibitor REYATAZ (atazanavir) and L-tert-leucine building block, among others. ${ }^{1}$ Cofactor $1,4-\mathrm{NADH}$ is very expensive ( $\$ 2600$ per mol) making its stoichiometric supply in a bioreaction not economically viable and necessitating its in situ regeneration. ${ }^{2}$ 1,4-NADH regeneration has been the area of extensive research over the past $\sim 40$ years with various methods having been investigated, including biocatalytic, electrochemical, chemical, photocatalytic, homogeneous catalytic, and heterogeneous catalytic. ${ }^{3}$

Besides the commercialised enzymatic method, other regeneration strategies, currently under development, may suffer from non-selective reduction of $\mathrm{NAD}^{+}$to a mixture of the enzymatically active 1,4-NADH, and its inactive 1,2-NADH and 1,6-NADH isomers $^{4}$ (see Scheme 1). These molecules absorb light at 340, 395 and $345 \mathrm{~nm}$, respectively. ${ }^{5}$ It is furthermore noteworthy that 1,2-NADH exhibits high instability in phosphate buffer solutions (PBS) at $\mathrm{pH} \leq 7$ with a life time of only $\sim 30 \mathrm{~min}^{6}{ }^{6}$ Considering these facts, it is surprising to note that a significant number of literature has been relying solely on UV-Vis at $340 \mathrm{~nm}$ to quantify 1,4-NADH concentration. ${ }^{7}$ Moreover, Morrison et al. ${ }^{8}$ stated that unusual situations (e.g. discrepancies in HPLC analyses of NADH isomers) were observed in isomers preparation. This may lead to

Chemical and Materials Engineering, School of Engineering, University of Aberdeen, Aberdeen AB24 3UE, UK. E-mail: xiaodong.wang@lancaster.ac.uk

$\dagger$ Electronic supplementary information (ESI) available. See DOI: 10.1039/ c9cc07805c

\# Present address: Department of Engineering, Lancaster University, Lancaster LA1 4 YW, UK. inaccurate or misleading conclusions, as other isomers were potentially not considered or distinguished. Another fact in NADH regeneration research is that the yield of 1,4-NADH (calculated based on the initial concentration of $\mathrm{NAD}^{+}$) is often reported, without determining $\mathrm{NAD}^{+}$conversion (i.e. with no evidence of $100 \%$ selectivity or not including a process materials balance). These limitations impose an extreme challenge in understanding the reaction mechanism for further optimisation. Given the significant recent interest and effort in this field (see Table S1, ESI $\dagger),{ }^{3,7}$ it is crucial that the concentrations of the products and unreacted reactant are unambiguously quantified.

We report here, for the first time, an analytical method that can track $\mathrm{NAD}^{+}$and both the desired and undesired forms of NADH using a combination of UV-Vis spectrophotometry and biological assays. The developed approach has been validated in a representative regeneration process.

In the course of a reaction, the reaction medium may consist of all four molecules as shown in Scheme 1. The most important indicator for catalytic performance and kinetic analysis is the conversion of $\mathrm{NAD}^{+}$and the selectivity to $1,4-\mathrm{NADH}$. We have accordingly customised an enzymatic assay kit to determine both $1,4-\mathrm{NADH}$ and $\mathrm{NAD}^{+}$. The kit works as an enzymatic

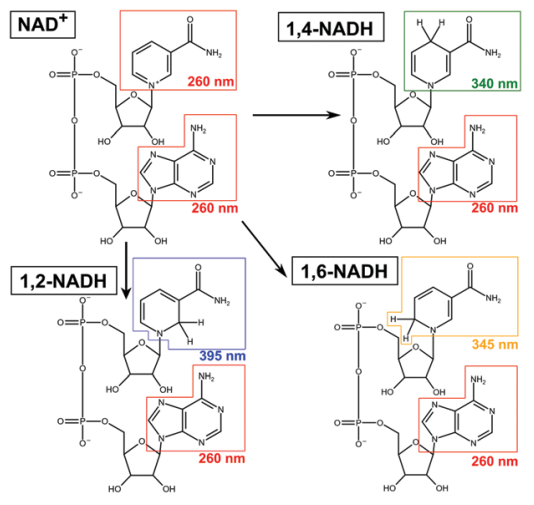

Scheme 1 Representative scheme for a non-selective NADH regeneration process in which $\mathrm{NAD}^{+}$reacts into 1,2-, 1,4- and 1,6-NADH. 

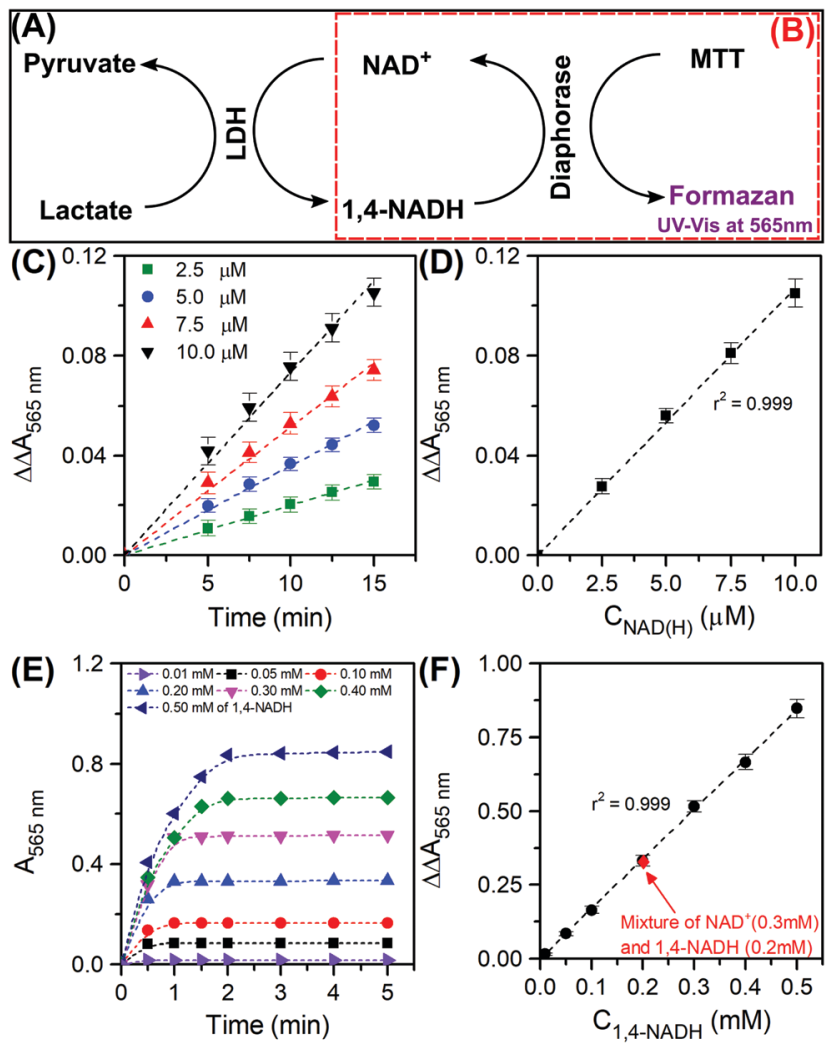

Fig. 1 Representative scheme of the reactions involved in the total enzymatic cyclic assay using both $\mathrm{NAD}^{+}$and 1,4-NADH (in black borders (A)) and the reactions involved in the enzymatic half cycle using 1,4-NADH only (in dashed red borders (B)); absorbance as function of time for the total cyclic assay for different $N A D(H)$ concentrations (2.5 to $10 \mu \mathrm{M})(\mathrm{C})$ and the corresponding calibration curve (D); absorbance as function of time for the half enzymatic cycle for different 1,4-NADH concentrations ( 0.01 to $0.5 \mathrm{mM})(\mathrm{E})$ and the corresponding calibration curve (F).

cyclic assay (Fig. 1A) in which lactate dehydrogenase (LDH) promotes the oxidation of lactate to pyruvate and $\mathrm{NAD}^{+}$ plays the role of the hydride acceptor, getting reduced to 1,4-NADH. The latter in turn releases the hydride to 3-(4,5dimethylthiazol-2-yl)-2,5-diphenyltetrazolium bromide (known as MTT, yellow colour) to be reduced by diaphorase to $(E, Z)-5-$ (4,5-dimethylthiazol-2-yl)-1,3-diphenylformazan (known as formazan, purple colour) that has a characteristic UV-Vis absorbance at $565 \mathrm{~nm}$ (Fig. 1B). The procedure presented here was specifically developed to be easily applicable to the various types of spectrometers available, not requiring a plate reader or other expensive analytical equipment. For this purpose, a working reagent was prepared by mixing $60 \mu \mathrm{L}$ of the assay buffer, $1 \mu \mathrm{L}$ of LDH, $1 \mu \mathrm{L}$ of diaphorase, $14 \mu \mathrm{L}$ of Lactate and $14 \mu \mathrm{L}$ of MTT. $80 \mu \mathrm{L}$ of the working reagent were added to $240 \mu \mathrm{L}$ of the $\mathrm{NAD}(\mathrm{H})$ (referring to the total amount of 1,4-NADH and $\mathrm{NAD}^{+}$, assay buffer was used as a diluent when needed). The mixture was thoroughly stirred very briefly, then transferred to a quartz cuvette $(2 \mathrm{~mm}$ path length, see ESI, $\dagger$ Part III for the detailed procedure and Fig. S1 and S2 for representative scans). The difference in absorbance at $565 \mathrm{~nm}$ between the sample (i.e. with cofactors) and blank (i.e. with no cofactors) was recorded with time using eqn (1) below:

$$
\Delta \Delta A_{\text {Sample }}=\Delta A_{\text {Sample }}-\Delta A_{\text {Blank }}
$$

where $\Delta A_{\text {Sample }}=A_{\text {Sample at time } t \text { min }}-A_{\text {Sample at o min }} ; \Delta A_{\text {Blank }}=$ $A_{\text {Blank at time } t \text { min }}-A_{\text {Blank at } 0 \mathrm{~min}}$. The difference in absorbance at $565 \mathrm{~nm}$ is directly proportional to the amount of $\mathrm{NAD}(\mathrm{H})$ in the system provided it stays linear with time. ${ }^{9}$ Fig. 1C shows the results of the kinetic measurements using our customised procedure which shows an excellent linear fit for the range of $\operatorname{NAD}(\mathrm{H})$ concentrations considered. Fig. 1D shows the calibration curve at $t=15 \mathrm{~min}$ for the total cycle and the resulting slope can be used for the quantification of the total amount of $\mathrm{NAD}(\mathrm{H})$ as follows:

$$
C_{\mathrm{NAD}(\mathrm{H})}(\mu \mathrm{M})=\frac{\Delta \Delta A_{\text {Sample }}}{\text { Slope }\left(\mu \mathrm{M}^{-1}\right)}=\frac{\Delta A_{\text {Sample }}-\Delta A_{\text {Blank }}}{\text { Slope }\left(\mu \mathrm{M}^{-1}\right)}
$$

With the concentration of $\operatorname{NAD}(H)$ known, if either of the two components can be quantified, the other can be directly determined. To determine the concentration of 1,4-NADH, we have innovatively used the reduction side (right half) of the kit as depicted in Fig. 1B, where 1,4-NADH reacts with MTT to form both $\mathrm{NAD}^{+}$and formazan. The working reagent was prepared by mixing $60 \mu \mathrm{L}$ of the assay buffer, $1 \mu \mathrm{L}$ of diaphorase and $14 \mu \mathrm{L}$ of the MTT. The whole working reagent $(75 \mu \mathrm{L})$ was then added to $240 \mu \mathrm{L}$ of $1,4-\mathrm{NADH}$ sample (in the range of 0.01 to $0.5 \mathrm{mM}$ ) and the absorbance was recorded with respect to time (see ESI, $\dagger$ Part IV for the detailed procedure). The kinetic results are shown in Fig. 1E. The absorbance increases rapidly and then stabilises. The absorbance at the plateau is an indication of the concentration of 1,4-NADH in solution and it was plotted against the actual concentration as seen in the calibration curve (Fig. 1F). Eqn (3) is used for the quantification of 1,4-NADH:

$$
C_{1,4-\mathrm{NADH}}(\mathrm{mM})=\frac{\Delta \Delta A_{\text {Sample }}}{\text { Slope }\left(\mathrm{mM}^{-1}\right)}=\frac{\Delta A_{\text {Sample }}-\Delta A_{\text {Blank }}}{\text { Slope }\left(\mathrm{mM}^{-1}\right)}
$$

The validity of the half cycle's novel use was tested by preparing a mixture of both $\mathrm{NAD}^{+}(0.3 \mathrm{mM})$ and $1,4-\mathrm{NADH}(0.2 \mathrm{mM})$. The absorbance measurement fit exceptionally well the calibration curve (see Fig. 1F), confirming that the existence of $\mathrm{NAD}^{+}$in solution has no practical effect on the 1,4-NADH analysis. The amount of $\mathrm{NAD}^{+}$is then deduced by subtracting the concentration of 1,4-NADH from the total $\mathrm{NAD}(\mathrm{H})$ using eqn (4):

$$
C_{\mathrm{NAD}^{+}}(\mathrm{mM})=C_{\mathrm{NAD}(\mathrm{H})}(\mathrm{mM})-C_{1,4-\mathrm{NADH}}(\mathrm{mM})
$$

To elucidate the reaction pathway and mechanism, knowing only the concentrations of $\mathrm{NAD}^{+}$and 1,4-NADH is not sufficient, unless the amount of reacted $\mathrm{NAD}^{+}$is equal to that of $1,4-\mathrm{NADH}$ produced, i.e. the reaction is $100 \%$ selective. When this is not the case, the key information to obtain is the concentrations of the NADH isomers produced, which is hampered by the fact that 1,4- and 1,6-NADH exhibit similar characteristic absorbance peak $(\sim 340 \mathrm{~nm})$ and 1,2-NADH decays quickly in PBS with $\mathrm{pH} \leq 7$ (which is commonly used in NADH regeneration). We have therefore developed a systematic approach to distinguish and quantify the three isomers. In order to quantify 
1,2- and 1,6-NADH in a reaction mixture, their molar absorptivities must be determined. Unfortunately, there is no supplier providing commercial isomer products (apart from 1,4-NADH). For this reason, we have synthesised a mixture of 1,2-, 1,4- and 1,6-NADH with known concentrations according to an established experimental procedure, ${ }^{10}$ i.e. sodium borohydride reduction of $\mathrm{NAD}^{+}$ (see ESI, $\dagger$ Part V). We conducted first the synthesis in PBS at $\mathrm{pH}=7$ as the high instability of 1,2-NADH at this condition (exhibits rapid decay) leaves only 1,4- and 1,6-NADH in the reaction medium.

In order to distinguish between the two isomers at $340 \mathrm{~nm}$, active $1,4-\mathrm{NADH}$ was reoxidised to $\mathrm{NAD}^{+}$by alcohol dehydrogenase $(\mathrm{ADH})$ catalysed acetaldehyde reduction ${ }^{11}$ (see the ESI, $\dagger$ Part VI). A reduction of almost half of the initial 340 absorbance was observed (Fig. 2A), in line with previous studies. ${ }^{10 c, 12}$ Upon 1,4-NADH oxidation, the absorbance at $260 \mathrm{~nm}$ increased dramatically indicating the formation of $\mathrm{NAD}^{+}$that has a high molar absorptivity at $260 \mathrm{~nm}$ compared to all isomers. ${ }^{5}$ The residual absorbance at $340 \mathrm{~nm}$ (due to 1,6-NADH) at five different initial concentrations of $\mathrm{NAD}^{+}$was then translated into a calibration curve of 1,6-NADH, which is depicted in Fig. 2B. A standard calibration curve for commercial 1,4-NADH at $340 \mathrm{~nm}$ was generated in the range of 0.05 to $0.3 \mathrm{mM}$. The slope of the linear curve corresponds to the molar absorptivity of the 1,4-NADH in $\mathrm{mM}^{-1} \mathrm{~cm}^{-1}$ (Fig. 2B).

In a course of a reaction, the absorbance of a sample at time $t$ at $340 \mathrm{~nm}, A_{340}$ (total), is a combination of both 1,6-NADH and 1,4-NADH absorptions. The latter can be determined by converting 1,4-NADH concentration (determined from the enzymatic half cycle) using $\varepsilon_{(1,4-\mathrm{NADH})}$ according to eqn (5):

$$
\begin{aligned}
A_{340(1,4-\mathrm{NADH})}= & \varepsilon_{1,4-\mathrm{NADH}}\left(\mathrm{mM}^{-1} \mathrm{~cm}^{-1}\right) \times L(\mathrm{~cm}) \\
& \times C_{1,4-\mathrm{NADH}}(\mathrm{mM})
\end{aligned}
$$

The absorbance and concentration of 1,6-NADH are then calculated using its molar absorptivity at $340 \mathrm{~nm}$ and the difference between the $A_{340 \text { (total) }}$ and $A_{340(1,4-\mathrm{NADH})}$ (see eqn (6) and (7)):

$$
\begin{gathered}
A_{340(1,6-\mathrm{NADH})}=A_{34 \mathrm{O}(\text { total })}-A_{34 \mathrm{O}(1,4-\mathrm{NADH})} \\
C_{1,6-\mathrm{NADH}}(\mathrm{mM})=\frac{A_{340(1,6-\mathrm{NADH})}}{\varepsilon_{1,6-\mathrm{NADH}}\left(\mathrm{mM}^{-1} \mathrm{~cm}^{-1}\right) \times L(\mathrm{~cm})}
\end{gathered}
$$
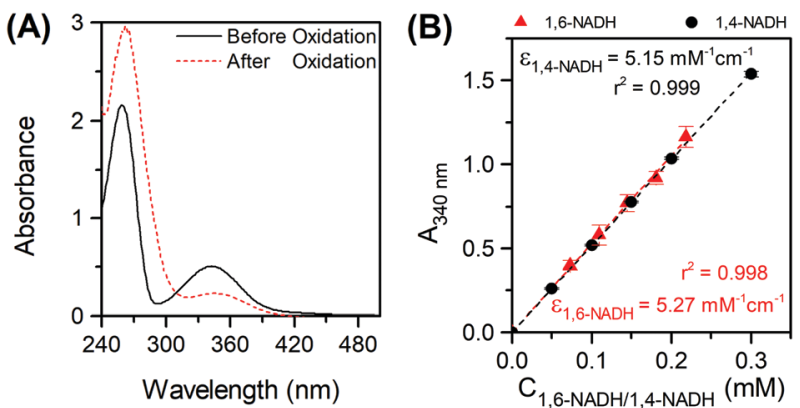

Fig. 2 Oxidation of a $\mathrm{NAD}^{+}$reduction mixture in phosphate buffer $\mathrm{pH} 7$ with acetaldehyde and $A D H$ with $C_{i}\left(N_{A D}{ }^{+}\right)=0.25 \mathrm{mM}(A)$ and 1,6- and 1,4$\mathrm{NADH}$ calibration curves at $340 \mathrm{~nm}(\mathrm{~B})$.
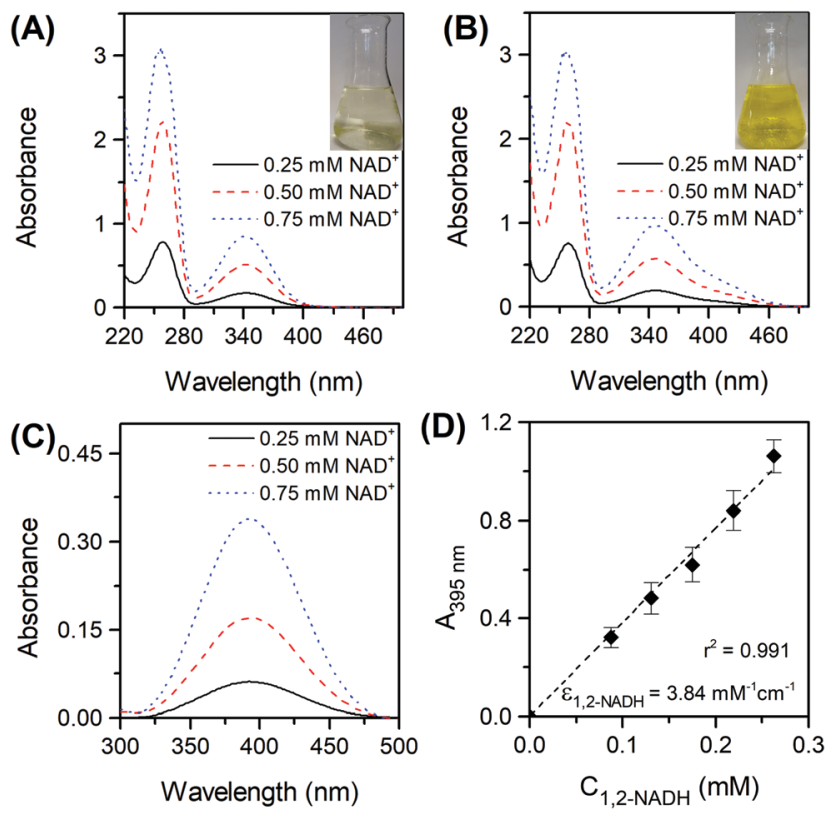

Fig. 3 Spectra of $\mathrm{NAD}^{+}$reduced by sodium borohydride in phosphate buffer $\mathrm{pH} 7$ (A) and in Tris buffer $\mathrm{pH} 8.5$ (B) at three different initial concentrations of $\mathrm{NAD}^{+}$(reaction conditions: $C_{i}\left(\mathrm{NAD}^{+}\right)=0.25-0.75 \mathrm{mM}$, $\mathrm{C}_{\mathrm{i}}\left(\mathrm{NaBH}_{4}\right)=70 \mathrm{C}_{\mathrm{i}}\left(\mathrm{NAD}^{+}\right), 22^{\circ} \mathrm{C}, 300 \mathrm{rpm}$, atmospheric pressure). Spectra difference obtained when spectra of the Tris-buffered reaction mixture was measured against the phosphate-buffered reaction mixture (C) and the corresponding 1,2-NADH calibration curve (D).

Fig. 3A presents the UV-Vis spectra of the products formed in the borohydride reduction of $\mathrm{NAD}^{+}$in $\mathrm{PBS}$ at $\mathrm{pH}$ 7. The reaction produced in the first few seconds a bright yellow mixture, which disappeared 1 minute later forming a very pale-yellow colour product (Fig. 3A inset). There is also no clear peak at $395 \mathrm{~nm}$, characteristic of 1,2-NADH, suggesting 1,2-NADH has decayed. ${ }^{10 b}$ In contrast, when $\mathrm{NAD}^{+}$was reduced in Tris buffer at $\mathrm{pH}$ 8.5, it generated a persistent bright yellow mixture with a wide spectrum between 300 and $480 \mathrm{~nm}$ (Fig. 3B). This vivid colour is associated with an absorbance band at $395 \mathrm{~nm}$, i.e. presence of 1,2-NADH. The difference spectrum between the two sets of reduction data (Fig. 3C) clearly shows the presence of the $395 \mathrm{~nm}$ absorbing species in Tris buffer. These results prove the impossibility to track the formation of 1,2-NADH in a PBS system at $\mathrm{pH} 7$. The difference in absorbance at $395 \mathrm{~nm}$ was then used for a calibration curve of 1,2$\mathrm{NADH}$ (Fig. 3D). It is important to mention that the $A_{395 \mathrm{~nm}}$ values in Fig. 3C are not identical to those in Fig. 3D because different dilution factors were used. The concentration of 1,2$\mathrm{NADH}$ can be calculated using the absorbance of the sample at $395 \mathrm{~nm}$ following eqn (8):

$$
C_{1,2-\mathrm{NADH}}(\mathrm{mM})=\frac{A_{395}}{\varepsilon_{1,2-\mathrm{NADH}}\left(\mathrm{mM}^{-1} \mathrm{~cm}^{-1}\right) \times L(\mathrm{~cm})}
$$

The validity of the proposed method was tested in the regeneration of $\mathrm{NADH}$ via the hydrogenation of $\mathrm{NAD}^{+}$(see ESI, $\dagger$ Part VII) using a commercial Pt/C catalyst, as previously reported. ${ }^{13}$ The concentration of each species was determined using the 

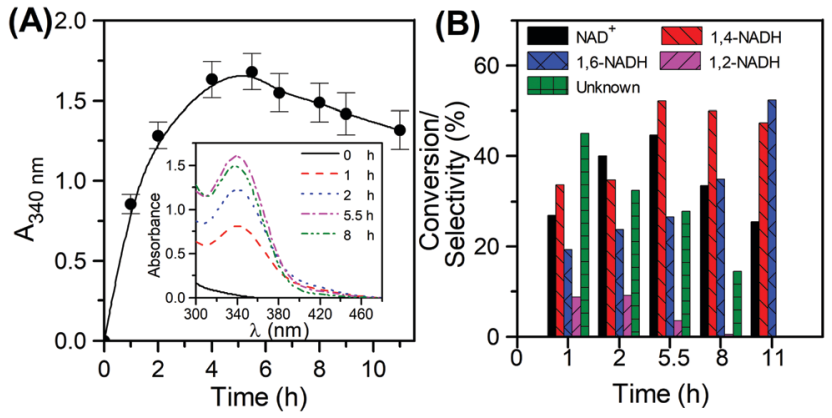

Fig. $4 \mathrm{NADH}$ regeneration using Pt/C. Evolution of the 340 absorbance with time (A), conversion of $\mathrm{NAD}^{+}$and selectivity of reaction products over time (B) (reaction conditions: $22^{\circ} \mathrm{C}, 8 \mathrm{~atm}$, Tris-buffer $\mathrm{pH} 8.5,900 \mathrm{rpm}$ and $C_{i}\left(N_{A D}^{+}\right)=1 \mathrm{mM}$

method described before, and the selectivity of each component was calculated using eqn (9):

$$
S_{\text {Compound,i }}(\%)=\frac{C_{\text {Compound,i }}(\mathrm{mM})}{C_{\text {Reacted } \mathrm{NAD}^{+}}(\mathrm{mM})} \times 100
$$

The reaction profile in the hydrogenation of $\mathrm{NAD}^{+}$was first tracked by measuring $340 \mathrm{~nm}$ absorbance with time (Fig. 4A). The scanned reaction mixtures (Fig. 4A inset) clearly show wide absorbance spectra, indicating the formation of all isomers. Based on Fig. 4B, the catalyst exhibits its maximum conversion at $5.5 \mathrm{~h}$. The analysis of the reaction mixture using the reductive cycle of the kit after $1 \mathrm{~h}$ reaction time, showed that only $60 \%$ of the total 340 absorbance amounted to the enzymatically active 1,4-NADH, with the remaining $40 \%$ being a contribution from 1,6-NADH. The colour of the sample taken at $1 \mathrm{~h}$ was slightly yellowish, but persistent, suggesting the formation of 1,2-NADH as well. This was confirmed by UV-Vis analysis at $395 \mathrm{~nm}$. The amount of reacted $\mathrm{NAD}^{+}$was determined using the total cycle of the kit by subtracting the concentration of $\mathrm{NAD}^{+}$left from the initial $\mathrm{NAD}^{+}$. Subtracting the concentration of all isomers $\left(1,2-, 1,4-\right.$, and 1,6-NADH) from the reacted $\mathrm{NAD}^{+}$resulted in a mole balance closure of $89 \%$ with the rest being attributed to unknowns. In order to understand the origin of these unknowns, it was important to perform a selectivity measurement of all components throughout the reaction. The results are shown in Fig. 4B. At the initial stages of the reaction (up to the first hour), high selectivity towards the unknown products is observed, followed by those of 1,4 , 1,6 and 1,2-NADH in decreasing order. As the reaction progresses and the conversion increases, the selectivity to the unknowns drops dramatically from $45 \%$ reaching eventually a zero value, while that of 1,4-NADH continues to rise reaching $52 \%$ at the maximum conversion). These results suggest that the unknown product formed may be an intermediate to the isomers rather than an ultimate by-product from $\mathrm{NAD}^{+}$hydrogenation. At the maximum conversion (45\%), the yields of 1,4-NADH, 1,6-NADH and 1,2-NADH were $23 \%$, $7 \%, 2 \%$, respectively. Using the $340 \mathrm{~nm}$ UV absorbance only (i.e. the method widely employed in the literature), the yield of 1,4-NADH would have been reported as $31 \%$, with an error of $35 \%$ with respect to the actual yield. This demonstrates the inaccuracy of using the $340 \mathrm{~nm}$ only method for 1,4-NADH quantification. It should be noted that the reported analytical method can also be used in other more complicated regeneration systems relying on sacrificial organic electron donors or hole scavengers (e.g. TEOA, see Fig. S3, ESI†).

In conclusion, we reported a method that uses UV-Vis spectrophotometry alongside biological assays for the determination of the concentrations of $\mathrm{NAD}^{+}$and both selective and non-selective compounds in NADH regeneration. The developed method, validated in a Pt/C catalysed regeneration reaction, can be applied to other NADH regeneration systems, providing critical information for mechanism/pathway analysis and further process optimisation.

This work was supported by The Royal Society (ICA|R1|180317 and IES $\backslash \mathrm{R} 3 \backslash 170162)$.

\section{Conflicts of interest}

There are no conflicts to declare.

\section{Notes and references}

1 C. Morrison, E. Heitmann, W. Armiger, D. Dodds and M. Koffas, Adv. Appl. Microbiol., 2018, 105, 51-86.

2 K. Faber, Biotransformations in Organic Chemistry, Springer, 2018.

3 (a) R. Wichmann and D. Vasic-Racki, Technology transfer in biotechnology, Springer, 2005, pp. 225-260; (b) H. Wu, C. Tian, X. Song, C. Liu, D. Yang and Z. Jiang, Green Chem., 2013, 15, 1773-1789; (c) X. Wang, T. Saba, H. H. P. Yiu, R. F. Howe, J. A. Anderson and J. Shi, Chem, 2017, 2, 621-654.

4 H. K. Chenault, E. S. Simon and G. M. Whitesides, Biotechnol. Genet. Eng. Rev., 1988, 6, 221-270.

5 H. Jaegfeldt, J. Electroanal. Chem. Interfacial Electrochem., 1981, 128, 355-370.

6 B. A. Beaupre, M. R. Hoag, J. Roman, F. H. Försterling and G. R. Moran, Biochemistry, 2015, 54, 795-806.

7 (a) H. Song, S. H. Lee, K. Won, J. H. Park, J. K. Kim, H. Lee, S. Moon, D. K. Kim and C. B. Park, Angew. Chem., Int. Ed., 2008, 47, 1749-1752; (b) S. Kim, G. Y. Lee, J. Lee, E. Rajkumar, J. Baeg and J. Kim, Electrochim. Acta, 2013, 96, 141-146; (c) X. Ji, C. Liu, J. Wang, Z. Su, G. Ma and S. Zhang, J. Mater. Chem. A, 2017, 5, 5511-5522; (d) D. Chen, D. Yang, Q. Wang and Z. Jiang, Ind. Eng. Chem. Res., 2006, 45, 4110-4116; (e) S. Kim, G. Y. Lee, J. Baeg, Y. Kim, S. Kim and J. Kim, J. Phys. Chem. C, 2014, 118, 25844-25852; $(f)$ K. E. Taylor and J. B. Jones, J. Am. Chem. Soc., 1976, 98, 5689-5694; $(g)$ L. Zhang, N. Vila, G. Kohring, A. Walcarius and M. Etienne, ACS Catal., 2017, 7, 4386-4394; (h) V. Ganesan, D. Sivanesan and S. Yoon, Inorg. Chem., 2017, 56, 1366-1374; (i) J. Canivet, G. Süss-Fink and P. Štěpnička, Eur. J. Inorg. Chem., 2007, 4736-4742; ( $j$ ) X. Huang, J. Liu, Q. Yang, Y. Liu, Y. Zhu, T. Li, Y. H. Tsang and X. Zhang, RSC Adv., 2016, 6, 101974-101980; (k) S. K. Kuk, R. K. Singh, D. H. Nam, R. Singh, J. K. Lee and C. B. Park, Angew. Chem., Int. Ed., 2017, 56, 3827-3832; ( $l$ ) D. H. Nam, S. K. Kuk, H. Choe, S. Lee, J. W. Ko, E. J. Son, E. G. Choi, Y. H. Kim and C. B. Park, Green Chem., 2016, 18, 5989-5993; (m) S. Wang, M. Li, A. J. Patil, S. Sun, L. Tian, D. Zhang, M. Cao and S. Mann, J. Mater. Chem. A, 2017, 5, 24612-24616.

8 C. S. Morrison, W. B. Armiger, D. R. Dodds, J. S. Dordick and M. A. Koffas, Biotechnol. Adv., 2017, 36, 120-131.

9 (a) H. Li, K. E. Worley and S. Calabrese Barton, ACS Catal., 2012, 2, 2572-2576; (b) Y. Gibon and F. Larher, Anal. Biochem., 1997, 251, 153-157; (c) C. Vilcheze, T. R. Weisbrod, B. Chen, L. Kremer, M. H. Hazbon, F. Wang, D. Alland, J. C. Sacchettini and W. R. Jacobs Jr, Antimicrob. Agents Chemother., 2005, 49, 708-720.

10 (a) M. B. Mathews, J. Biol. Chem., 1948, 176, 229-232; (b) S. Chaykin and L. Meissner, Biochem. Biophys. Res. Commun., 1964, 14, 233-240; (c) S. Chaykin, L. King and J. G. Watson, Biochim. Biophys. Acta, 1966, 124, 13-25.

11 S. Chaykin, K. Chakraverty, L. King and J. G. Watson, Biochim. Biophys., 1966, 124, 1-12.

12 M. B. Mathews and E. E. Conn, J. Am. Chem. Soc., 1953, 75, 5428-5430.

13 X. Wang and H. H. P. Yiu, ACS Catal., 2016, 6, 1880-1886. 